\title{
Online monitoring of essential components helps urban transport management and increases the safety of rail transport
}

\author{
A. Daadbin ${ }^{1}$, J. Rosinski ${ }^{2} \&$ D. Smurthwaite ${ }^{2}$ \\ ${ }^{1}$ School of Computing Engineering \& Information Sciences, \\ Northumbria University, UK \\ ${ }^{2}$ Transmission Dynamics, UK
}

\begin{abstract}
Complex modern transport systems need to be reliable and operated at high efficiency with the cost of breakdowns kept at a minimum. The well known strategy for maintaining the operation of any integrated system is to choose the appropriate parameters to be monitored continuously. Then, any fault or deviation from the normal operation condition would be detected and the necessary action could be taken to avoid any disruption to the operation and avoid any accident with devastating consequences.

This paper looks at monitoring of parameters for essential components in rail transport such as axle vibration and pantograph load, and gives the detail of the systems used in monitoring their operation. The continuously measured data such as torque on the main gearbox shaft or load/acceleration on the pantograph enables diagnosis of potential issues within such systems on routinely operating trains. Variations from the expected values can be detected early and corrected to avoid compromising passenger safety from a more severe failure in the future.

The paper discusses the system designed for monitoring the transmitted torque and bending moments in the rail vehicle axle as a mean for identification of any abnormal loading.

Pantograph failures due to complex interactions between the overhead line (OHL) and pantograph structure cause significant problems to railway industry worldwide.

This paper also describes the design, development and test results from the first fully proven Pantograph Monitoring System which is now deployed on routinely operating trains in the UK.
\end{abstract}


High alarm events initiated from any component being monitored are instantaneously transferred to the train to warn the operator and the control centre about potentially harmful event which require immediate attention. This will in turn increase safety of the travelling passengers from any consequences of developing failures.

Keywords: flat wheel, bogie gearbox, axle vibration, pantograph failure, monitoring system, condition monitoring, Bluetooth communication.

\section{Axle vibration}

The development in the rail technology has created a relatively safe and economical transport system. Parallel with this progress in rail technology some health monitoring techniques have been developed and refined. Typically, vehicle health monitoring is designed in during the early stages of development to increase system availability by reporting vehicle operating health and specific subsystem faults to the train operator and central control room [1]. For locomotives using diesel engines, various systems have been developed to collect data and diagnose faults with the aim of reducing vehicle maintenance [2].

For detection of faults on rail vehicles, an interacting multiple-model algorithm has been used [3] where simulation studies have been completed and field verification has been carried out. Later work [4] has developed a portable condition monitoring system for use on in-service vehicles. The system is able to estimate the irregularities of the rails from vertical and lateral acceleration of car body. A GPS system is also used together with to localise the fault on track and monitor developing faults.

Early work carried out in electrically noisy rail traction systems highlighted difficulties with torque measurements on the motorised axle sets resulting from significant Electromagnetic Interference (EMI). These initial difficulties prompted the development of miniature strain gauge amplifiers which can be positioned very close to the gauges, avoiding EMI caused by longer strain gauge wiring runs, and miniature telemetry systems designed to cope with the demanding environment.

These miniature strain gauge amplifiers and FM telemetry oscillators are a fully configured self-contained system, with stabilised strain gauge supply, strain gauge amplifier, voltage-to-frequency converter and a short range FM transmitter, which include on-board filters designed for rejection of EMI. These systems can transfer FM data using capacitive, inductive and Infra-Red signal transmission. The main concept behind using these miniature systems is to install them in very close proximity to the strain gauges, thus reducing noise pick-up from longer connecting wires. It is noted that the small size of these systems (size of the complete PCB is similar to the size of the average strain gauge) allows a very compact and integral installation. A typical application of a short range telemetry system installed on a rail axle is shown in Figure 1.

In a typical application the data loggers are programmed using a PC and are set to operate unattended for periods up to 4 years of continuous operation. Over 


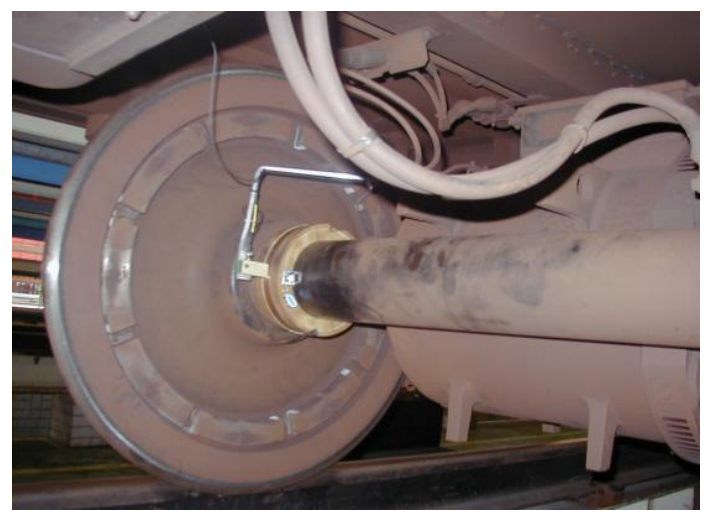

Figure 1: Short range FM telemetry installed on a motorised axle - Singapore.

recent years the size of the loggers has been significantly reduced, while their capabilities have increased. The overall size of today's data logger is 50x30x12 $\mathrm{mm}$, which makes it ideal for application in restricted places and on rotating machinery. An example of an uncased Radio Microlog with Rainflow and Time at Level analysis capability, and 100x4 sec of highest signal time domain data storage (4 Mb on board memory capacity) with Bluetooth telemetry system for data downloading and radio communication as shown in Figure 2.

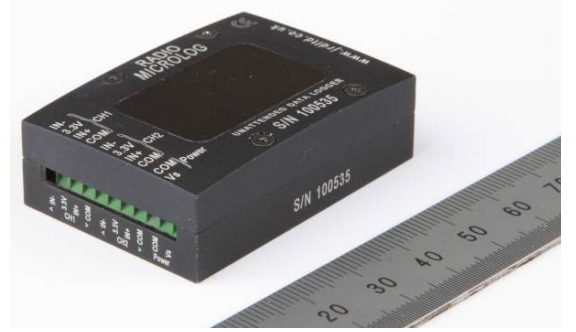

Figure 2: $\quad$ Miniature data logger with Bluetooth communication.

The data loggers are now so small that in practice the size of the final installation is governed entirely by the size of power supply. When space is not critical and access is not restricted a battery operated system will offer a robust and practical solution. An example of such a system installed on a propeller shaft of a commercial vehicle is shown in Figure 3 below.

When batteries cannot be easily changed or the system requires permanent power, an inductive power supply is recommended. A typical application showing a data logger powered by an inductive loop is shown in Figure 4. This photo shows the long-term data logger installation on the main torque tube of a $6 \mathrm{MW}$ Gas Turbine powered generator, where the shaft runs at 1500 RPM. 


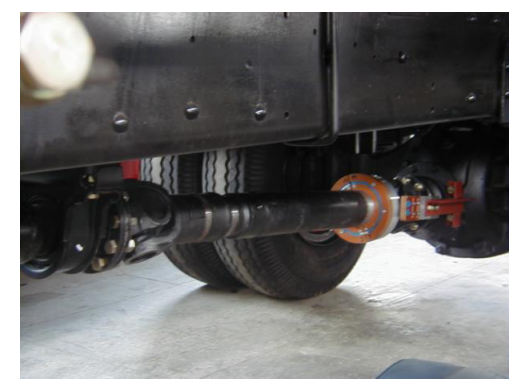

Figure 3: An example of battery powered data logger fitted on a propeller shaft (shaft speed 4200 RPM, battery life 3 months, temperature range $-30^{\circ} \mathrm{C}$ to $\left.+60^{\circ} \mathrm{C}\right)-\mathrm{China}$.

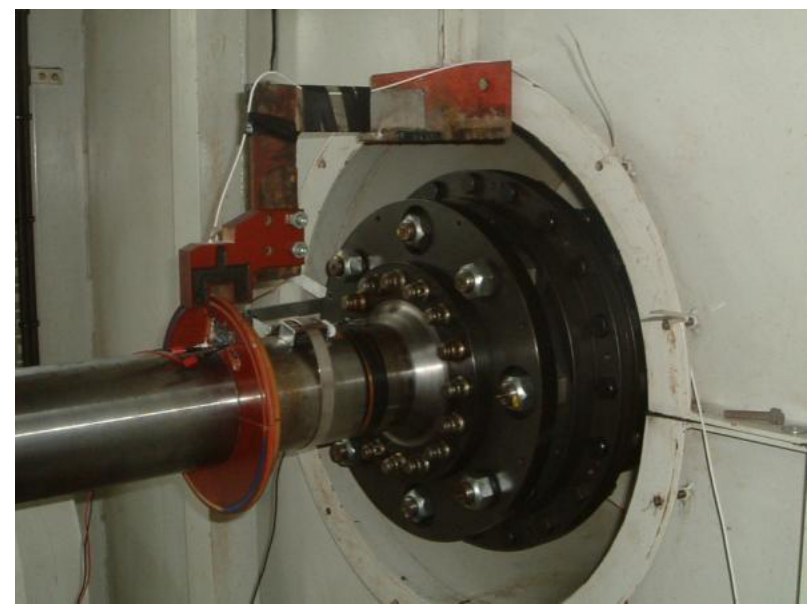

Figure 4: An example of inductively powered data logger fitted on a $6 \mathrm{MW}$ generator torque tube - Bangladesh.

Inductive power supplies of this type have been used successfully at shaft speeds up to 25,000 RPM.

The miniature data loggers have been further developed with a fast integral digital communication link (Bluetooth) so that up to six loggers can be monitored and controlled from a CPU, which has GPS and GSM capability. This allows for the simultaneous recording of time domain data on all loggers when an extreme signal is detected by any one. With the GPS facility, the location of each extreme event (which is recorded as time domain data) is also recorded. Finally, all these systems are re-programmable by radio communication making them extremely flexible in case of future software updates.

These miniature data loggers have been successfully used in railway applications, not only to acquire axle stress data but also to correlate this data with axle box acceleration, axle load, vehicle speed and train location on the 
track. A comprehensive Rail Axle Stress Monitoring (RASM) system typically consists of:

- Two axle stress data loggers fitted on one axle, measuring bending stress in two planes, and torsional axle stress (3-channel data logging)

- Four 2-axis acceleration data loggers fitted on each axle box on one bogie

- Radio transceiver unit fitted centrally on a bogie, synchronising operation of six data loggers and the Central Processing Unit (CPU)

- $\quad$ CPU fitted in a convenient location within the vehicle, equipped with memory storage device (128 Mb MMC card), on board GPS system to accurately locate "high" events (optional $2 \mathrm{~m}$ or $15 \mathrm{~m}$ positional accuracy) and GSM engine for continuous mobile network link (SMS messaging, uploading critical data, emergency calls, etc.)

A schematic view of the complete system is shown in Figure 5.

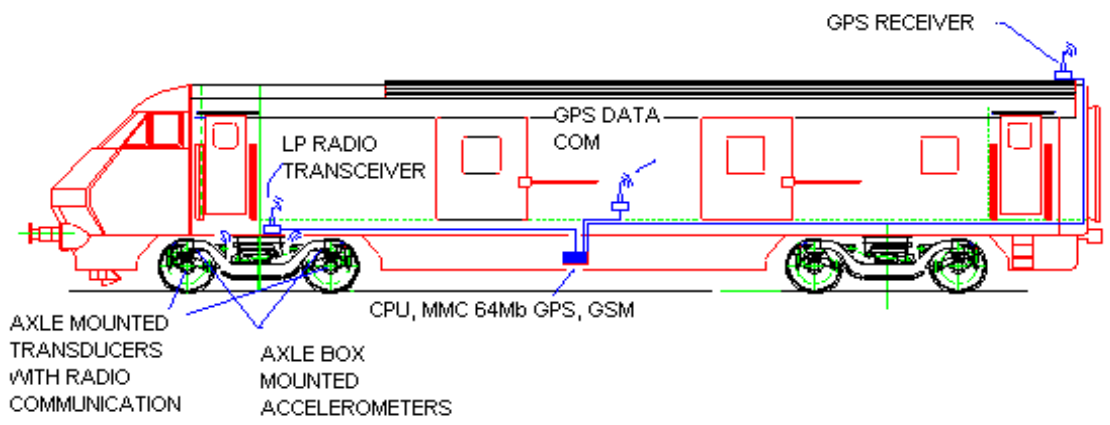

Figure 5: Rail axle stress monitoring system designed for application on routinely operating trains.

Each of the six data loggers is programmed not only to perform Rainflow Count (counting fatigue or acceleration cycles) but also to acquire 100 highest events in the time domain, synchronised on all channels. By logging synchronised data from axle stress and axle box acceleration it is possible to correlate high axle stress events with vehicle operation and to study dynamic wheel - rail interaction.

With the rail axle data logging systems fitted on routinely operating trains it is possible to monitor critical sections of the track and to effectively determine track deterioration. Such data will greatly improve our understanding of axle stressing, and how this correlates with axle acceleration and track features. It provides useful data for both basic academic work, such as wheel-rail interface modelling, and also facilitates the development of more rational track maintenance criteria based on axle stress and wheel set acceleration limits.

A typical signal showing the bending stress on the shaft is shown in Figure 6 for a 'crush laden' car, loaded as shown in Figure 7. 


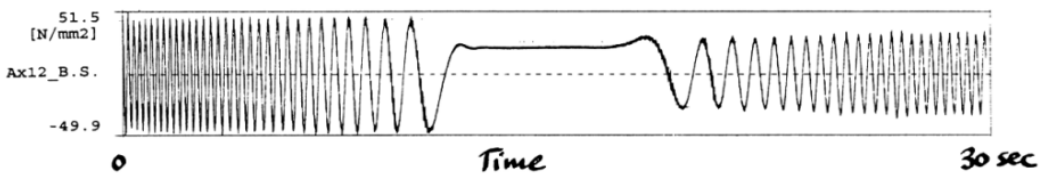

Figure 6: Axle bending stresses measured for a crush laden motor car MC2 during E/D braking followed by acceleration at notch 7 in the same direction (axle No 11 leading).

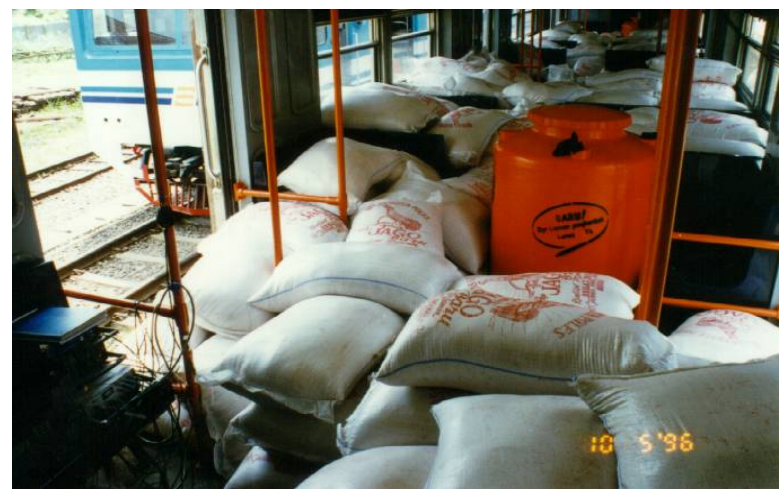

Figure 7: Crush laden car.

To evaluate a better understanding of the shaft behaviour during the power/torque transmission both bending stress [top trace] and torsional stress [bottom trace] are recorded. A typical signal is shown in Figure 8.

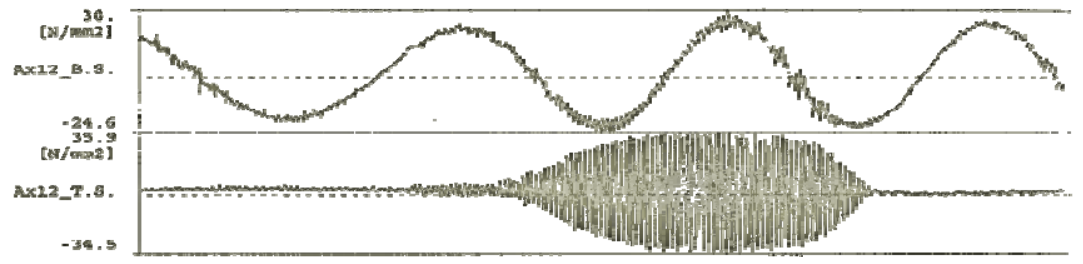

Figure 8: Bending and torsional stresses measured simultaneously on axle No 12 during self-excited axle vibration.

\section{Pantograph loads}

Pantographs are the single contact point between the rolling stock and the catenary. Good contact must be maintained under all running conditions to 
ensure the seamless collection of electrical power. The higher the speed, the more difficult it is to maintain good contact.

In Europe the overhead line infrastructure is designed for a lifespan of 30-50 years plus. This has resulted in the selection of specific materials such as pure carbon or copper and graphite impregnated carbon for the critical pantograph contact strips. However, these materials present the drawback of wearing very rapidly, increasing the need for intense regular maintenance.

Traditionally European railways support a maintenance strategy based on inspecting and replacing pantograph heads rather than focusing on the overhead infrastructure. Problems with the overhead line during contact with the pantograph strip can promote wear and damage to the pantograph carbon element. There are reports of pantograph heads needing replacement after a single journey on high speed trains. Therefore, a monitoring system for accurate identification of the overhead line geometry faults and their locations is extremely valuable.

The background and state-of-the-art in pantograph monitoring is discussed in [5]. To ensure the seamless collection of power, pantographs must maintain good contact with Overhead Line under all running conditions. The unstable current collection condition on the overhead line caused by contact force fluctuation has been studied in [6]. This study confirms that the contact force amplitude has a correlation with conditions of the height profile of contact wires and the contact wire mechanical faults.

Due to the nature of the environment in which the Pantograph operates, it is very difficult to put any monitoring in place. Figure 9 show the role of the pantograph in connecting the supply from the overhead line to the train.

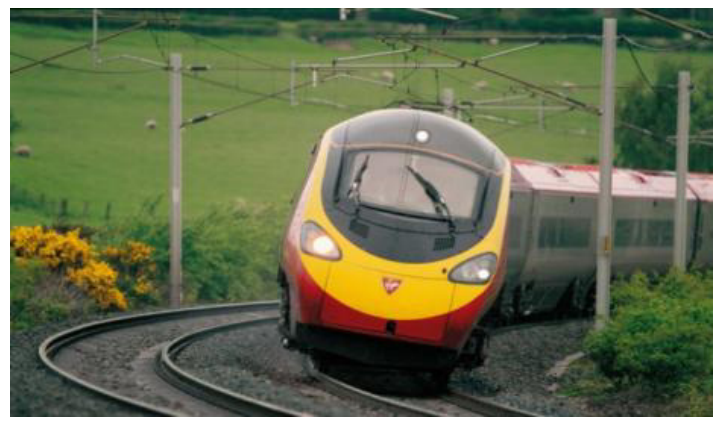

Figure 9: Pantograph connecting overhead line to the train.

Taking measurements of the interaction between train pantograph and the overhead line catenary has long been a challenge; the principal obstacle is to overcome the hostile environment and the problems of isolation. Such measurements require a telemetry system to transmit information from pantograph-mounted transducers, at a potential of $25 \mathrm{kV}$, to recording equipment located in the vehicle body. Transmission Dynamics in conjunction with Serco 
Rail has developed and successfully implemented a Pantograph Monitoring System which is now deployed on routinely operating trains in the UK.

In Figure 10 the close-up view of the pantograph in the lowered position is shown. When the power is needed, the mechanism is raised until the carbon strips get closer to the overhead line making contact. The Digital Processing Module (DPM) is directly mounted on the pantograph structure in the foreground, and the three photovoltaic cells which supplement the on-board battery power supply can be clearly seen. The DPM is shown in greater detail in Figure 11.

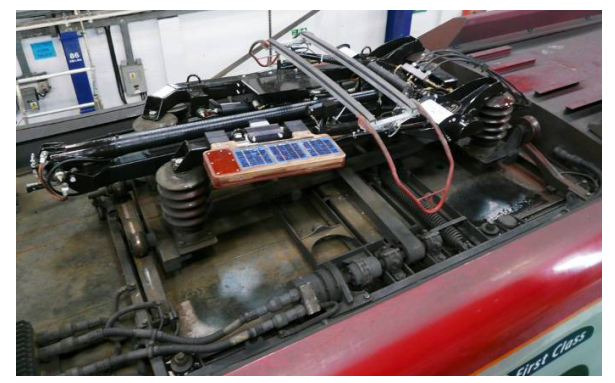

Figure 10: $\quad$ Pantograph high EMC, Arcing $25 \mathrm{kV}$ life f.

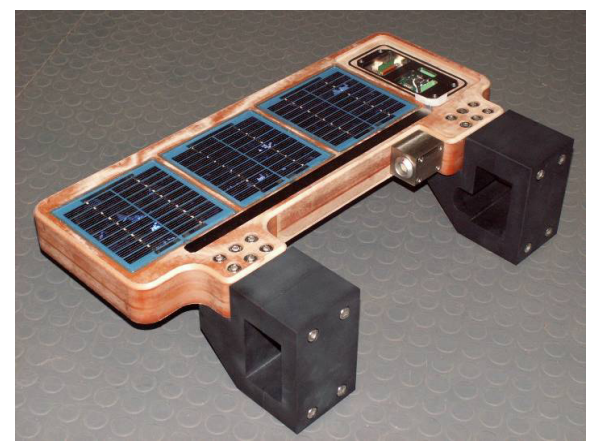

Figure 11: Digital processing module.

The Pantograph Damage Assessment System (PANDAS) features are:

- Directly mounted on a $25 \mathrm{kV}$ live pantograph

- Bluetooth radio communication with train

- $\quad$ On Board GPS for accurate event location stamp

- $\quad$ Battery / Solar PV Panel Powered - long operation life

- On board data storage and signal processing

- Use of two accelerometers and two 'dummy' channels

- Overnight download of all events to train-mounted SD memory card

- Connected to GPRS mobile network for immediate access and interrogation 
- Remotely re-programmable via mobile network

- Robust design - Proven in Service

- $\quad$ EMI certified for operation in the demanding rail environment

The typical signal from the two accelerometers on the carbon strip is shown in Figure 12. It shows the acceleration caused by the impact load on the pantograph.

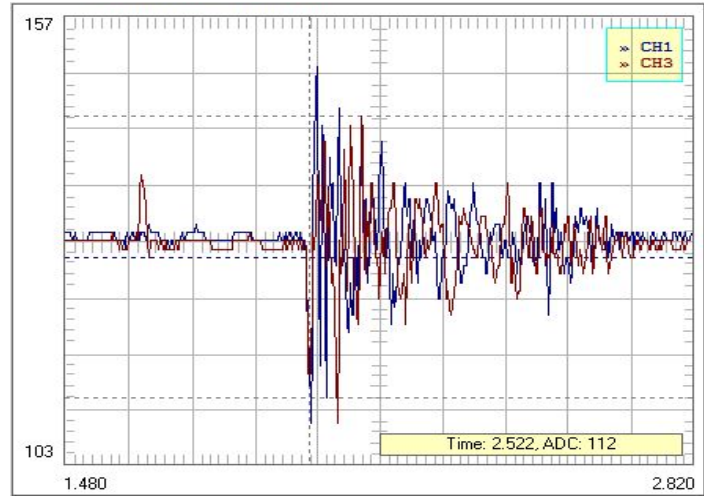

Figure 12: Signal from the accelerometer.

The instantaneous monitoring enables the 'hot spots' caused by overhead line to be mapped and trended to allow successful implementation of predictive maintenance of the Over-Head Line (OHL), Figure 13.

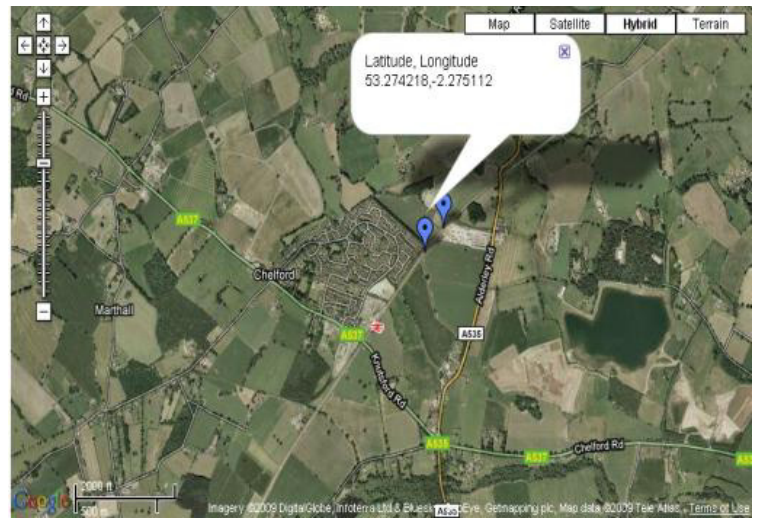

Figure 13: OHL fault location stamp.

The system is programmed to calculate the location of the event, allowing for the slight delay between the detection of an actual triggered event and the receipt 
of the next GPS signal. The improved accuracy obtained by carrying out this adjustment can be seen in Figure 14.

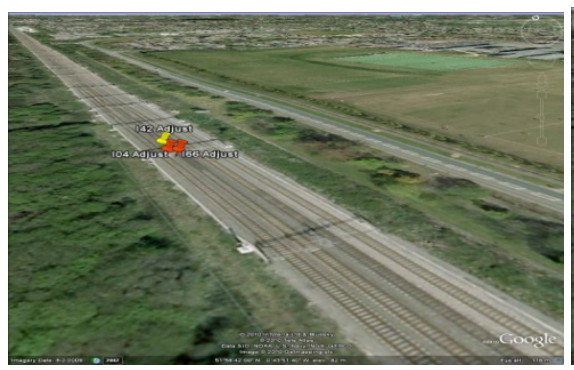

Initial position for two logged events

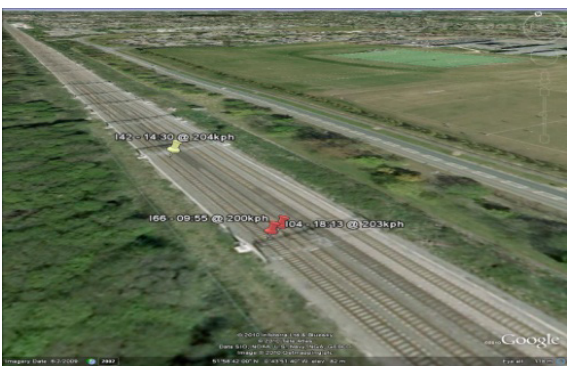

Adjusted location of events

Figure 14: Enhanced accuracy of location for triggered events.

Events shown in Figures 15 to 17 illustrate how an OHL line defect was detected at the same location on three separate occasions on the same day, by trains travelling at approximately $200 \mathrm{~km} / \mathrm{h}$.

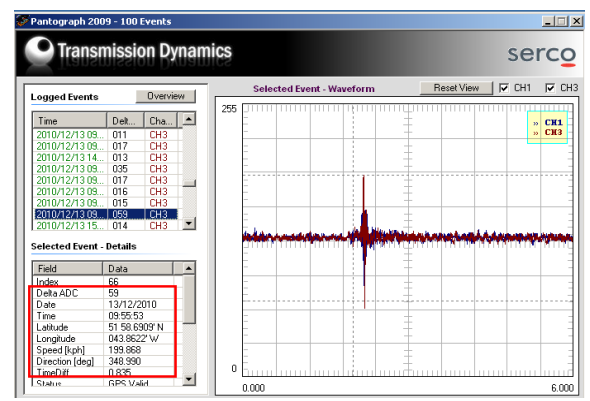

Figure 15: Event 1.

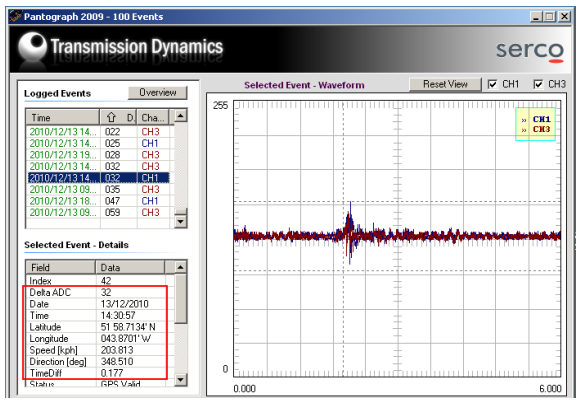

Figure 16: Event 2. 


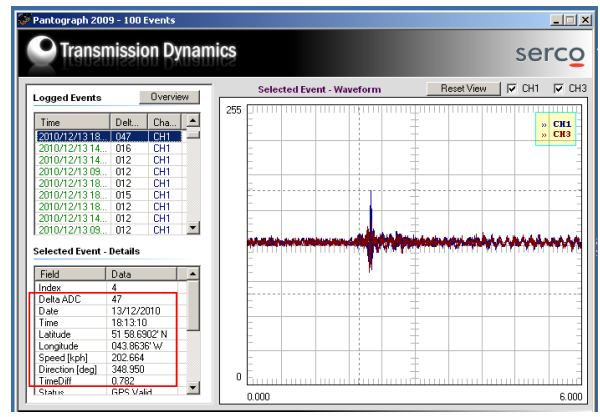

Figure 17: Event 3.

The above described Pantograph Damage Monitoring System (PANDAS) has been in continuous service and has been awarded 2010 UK Rail Business for Engineering Excellence. One of the main advantages of this system is totally unmanned Data Automation where the fault is instantaneously notified by a full report and emailed, confirmed by SMS, to designated recipients.

\section{Conclusions}

The development of strain gauge instrumentation, telemetry systems and data loggers for demanding transport applications is illustrated by multiple examples showing implementation of miniature data loggers and strain gauge instrumentation on a wide range of components on the train. Based on this experience a modern Rail Axle Stress Monitoring system was developed for application on the routinely operating trains.

The Pantograph Damage Assessment System (PANDAS) described in this paper also represents the newest developments in pantograph monitoring and it is now in routine operation on the Class 390 'Pendolino' tilting trains operating on the West Coast Main Line (WCML) in the UK. The system obtained Network Rail acceptance and is being deployed across the UK. The main features Bluetooth radio communication with train and on-board GPS enable the rapid and accurate identification of the location and exact time and date at which significant pantograph events occurred. A comprehensive report is automatically created and distributed by e-mail and SMS.

This innovative monitoring system reduces the maintenance costs not only for the pantograph but also for the overhead line electrical equipment.

\section{References}

[1] McDonald M, Richards A, Vehicle health monitoring on the docklands light railway. Advanced Condition Monitoring Systems of Railways, IEE Colloquim on $4^{\text {th }}$ October 1995 , Vol 4, pages 1-6. 
[2] Preston, K S, Vehicle systems monitoring technology at BR Research. Advanced Condition Monitoring Systems of Railways, IEE Colloquim on $4^{\text {th }}$ October 1995. Vol 3, pages 1-6.

[3] Hayashi Y, Kojima T, Tsunashima H, Marumo Y, Real time fault detection of railway vehicles and tracks, Railway Condition Monitoring 2006, The Institution of Engineering and Technology, pages 20-25.

[4] Tsunashima H, Kojima T, Marumo Y, Matsumoto A, Mizuma T, Condition monitoring of railway track and driver using in-service vehicle. $4^{\text {th }}$ IET International Conference on Railway Condition Monitoring 2008, pages 1-6.

[5] O'Donnell C, Palacin R, Rosinski J, Pantograph damage and wear monitoring system, Condition Monitoring 2006, The Institution of Engineering and Technology, pages 178-181.

[6] Harada S, Shunichi K, Monitoring of overhead contact line based on contact force, Railway Condition Monitoring 2006, The Institution of Engineering and Technology, pages 188-193. 